\title{
The psychotherapy department and the community mental health team: bridges and boundaries
}

\author{
Jeremy Holmes
}

\begin{abstract}
Aims and method The aim of the study was to clarify the role of psychotherapy departments in relation to a general psychiatric service, and in particular community mental health teams (CMHTs). Literature reviews of therapeutic activities in CMHTs and of psychotherapy delivery methods were undertaken. The implications of the National Health Service Executive document on Psychotherapy Services in England was considered, based on experience of the psychotherapy-general psychiatry interfaces in North Devon and Bristol.
\end{abstract}

Results Psychological therapies are an integral part of psychiatric treatment provided in CMHTs, but are often delivered without careful assessment, training or supervision. Psychotherapy departments contain expertise in the range of psychological therapies, but are sometimes percelved as remote from everyday psychiatric practice. Ways in which the divide between general psychiatry and psychotherapy might be bridged are suggested. A multi-disciplinary psychological treatment unit can offer specialist resources for the assessment and treatment of complex cases, especially those with personality disorders; psychological interventions in psychosis; and brief focused therapies for neurotic disorders unresponsive to drugs.

Clinical implications Consultation and liaison with the CMHT should become a key element in the work of a psychological treatment unit, and structured therapies under supenvision similarly central to the work of CMHTs.

\section{Psychotherapy Services in England Report}

A Government-commissioned survey of NHS psychotherapy services in England (National Health Service Executive, 1996) showed that the bulk of psychotherapeutic work is not carried out by qualified psychotherapists and does not take place in psychotherapy departments. It is delivered by general mental health professionals in a variety of settings: for example, counsellors working in general practice or community psychiatric nurses (CPNs) based in community mental health teams (CMHTs). The report proposes a classification of psychotherapies which takes account of this. 'Type $A^{\prime}$ psychotherapy is that most likely to be found within CMHTs as part of a general package of care of which psychotherapy is but one component. Type B and $C$ therapies are more specialised eclectic or model-based therapies, typically delivered, respectively, within psychology or psychotherapy departments.

The report is critical of the organisation, quality control and efficacy of much of what currently passes for psychotherapy. It proposes a central role for psychotherapy departments or psychological treatment units (PTUs) in improving the coordination and delivery of safe, effective, equitable and accountable psychotherapy. It argues that psychotherapy should, through training and supervision, become pait of mainstream psychiatric treatment, and encourages PTUs to become multi-modal rather than exclusively psychoanalytic.

\section{Community mental health team}

In many mental health units, general psychiatric work is centred on the CMHT. Such units frequently operate a 'single point of entry' system in which referrals are scrutinised by a coordinator and then allocated as appropriate to team members, CPN, psychologist, occupational therapist or psychiatrist, for assessment. The result of that assessment is then fed back to a multi-disciplinary team, and following Government guidelines (National Health Service Executive, 1994) each case is then prioritised (according to the Care Programme Approach (CPA) 'level') and allocated to a keyworker, who supposedly coordinates the various services and treatments required. 
In practice, this system can be problematic in two main ways, the first is the sheer bulk of referrals which the team may receive. This can lead to enormously heavy case loads for CMHT workers, who can do no more than offer minimal support to many of their clients. Second, there is often lack of clarity about the nature of the psychotherapeutic input required by particular clients. The CMHT assessment is not informed by specific psychotherapy assessment skills, and patients may either inappropriately be offered Types B or C treatments by untrained therapists, when a less intensive supportive approach is indicated; or conversely may offer vague support' for want of relevant training or targeted psychotherapy services.

\section{Psychological treatment unit}

Specialist psychotherapy services are unevenly distributed across the country. Some districts have no consultant psychotherapist, despite a Royal College of Psychiatrists' recommendation, first endorsed 20 years ago, that there should be a minimum of one consultant psychotherapist per 200000 of the population (Royal College of Psychiatrists, 1991). District psychology services are generally better established, providing psychotherapeutic input into mental health units, liaison psychotherapy services to medical and surgical departments, and sometimes sessional work in general practitioners' surgeries. However, waiting times for treatment are often very long, and treatment is not necessarily directed towards those with severe mental illness.

Relationships between psychology and psychotherapy services vary from the distant, through cordial cooperation, to rare instances of complete integration into a psychological treatment unit. Psychotherapy departments have tended to be predominantly psychoanalytic in orientation, while psychology departments are more likely to specialise in cognitive-behavioural (CBT) treatments. Referrals to both come directly from general practitioners, and internally from the CMHTs.

Relationships with CMHTs vary. Psychologists may be members of the team, usually on a parttime basis. Psychotherapy departments have traditionally had no direct links with the CMHTs, but there is an increasing trend for members of the PTU to devote sessional time to the CMHT, offering assessment of difficult cases and supervision for team members. Sometimes the CMHTpsychotherapy relationship is centripetal, with CMHT workers seconded to the psychotherapy department for one or two sessions per week.

Following an intensive assessment interview, often backed up with self-report questionnaires, psychotherapy departments generally decide whether referred patients are 'suitable' or not for psychotherapy, allocate them to a waiting list, from which they are taken on in due course, frequently by a junior therapist under the supervision of a senior member of the team. As with CMHTs, appraisal of 'suitability' is limited by the therapy options available. If analytic therapy is the predominant model, then the criteria for analytic therapy will determine the outcome of assessment, if it is counselling and short-term groups, then counselling and short-term groups is what clients will be offered, and so on.

Recently some departments have attempted to move away from the rather narrow focus thus far described. The psychology-psychotherapy rapprochement characteristic of PTUs has the advantage of combining psychoanalytic, CBT and sometimes systemic skills, thereby offering patients a greater range of psychotherapeutic options, and broadening the usefulness of the PTU to the mental health unit as a whole. A step further moves psychotherapy away from modelbased treatment towards targeting specific disorders. For instance, a PTU may offer a personality-disorder service, sometimes combined with a psychotherapy day hospital (Bateman, 1997). Psychotherapy may be offered to those suffering from early trauma (usually sexual abuse), or as a central strand in services for those with eating disorders, resistant depression, recurrent deliberate self-harm, first episodes of psychosis or sexual disorders. Here the modality of therapy is tailored to the needs of the patient and the illness, and can more easily form part of an evidence-based pattern of treatment (Roth \& Fonagy, 1996).

\section{New patterns of service: the interdependence of the PTU and the CMIHT}

How can these two areas of mental health work - that of the CMHT and the PTU - the one containing enthusiastic but uncoordinated potential, the other a repository of expertise but often sequestered from the hurlyburly of general psychiatry, be brought more creatively together? How can proven effectiveness be translated into clinical efficacy? An earlier paper advocated a 'hub and spokes' arrangement in which a multidisciplinary, multi-modal PTU retained a critical central mass of psychotherapeutic expertise. while reaching out to the rest of the mental health unit (Holmes \& Mitchison, 1995). This paper builds on that model, but suggests how it can be interlocked with a more structured pattern of psychotherapeutic work within the CMHT. 
The National Health Service Executive (1996) document emphasises the need for services to evolve in response to local needs, skills and enthusiasms. Nevertheless current trends suggest a number of guiding principles which can inform the relationship between CMHTs, PTUs, and primary care.

\section{The PTU}

The prime role of the PTU is to be a repository of psychotherapeutic expertise offering training, supervision, assessment and treatment of complex cases to the CMHTs. The PTU is explicitly multi-disciplinary and divides its work along two dimensions. The first is the traditional modelbased one, and enables members to offer training, supervision, and treatment in the various modalities (e.g. family therapy, CBT, psychoanalytic).

At the same time, expertise in diagnosis-based therapies needs to be developed. Here too local needs and expertise will determine patterns of service, but, as a minimum, three main areas of expertise need to be developed: (a) psychological interventions in psychosis, as part of an early intervention in psychosis programme: (b) specific treatments for complex cases of anxiety/depression, usually with time-limited therapies; (c) a service for patients with personality disorder, with especial emphasis on selection of cases suitable for intensive longer-term therapy. Other possible foci might include eating disorders and somatisation disorders.

Each division is headed by a senior member of the PTU. There will be a tendency for CBT and systemic therapists to be drawn to the psychosis and anxiety/depression teams, while analytic therapists may focus more on the longer-term treatment of patients with personality disorder, but no single approach is exclusively effective, and integrative treatments need to evolve (Albeniz \& Holmes, 1995). Members of the PTU will be organised in a number of sub-units, each of which with its own identity, but which meet regularly together for audit, case discussion and other academic work.

\section{The CMHT}

In numerical terms the majority of psychotherapeutic work within a mental health unit will be carried out in the CMHTs. There is an urgent need for improved organisation, audit and enhancement of psychotherapeutic skills within the CMHT.

After assessment, cases referred to the CMHT are assigned to a number of categories: (a) refer back to primary care, no further intervention needed; (b) refer to early intervention in psychosis team; (c) refer to brief intervention team for focused work for mild/moderate depression or anxiety (e.g. anxiety management or self-esteem group, CBT, focal dynamic counselling/therapy); (d) refer to long-term therapy team for patients with personality disorder, and/or early trauma; (e) intensive psychotherapeutic intervention not required, assign to supportive therapy at low intensity.

As with the PTU, each member of the CMHT has a dual role. Members divide their work between assessment and carrying a long-term case load as at present, but are also part of a specific psychotherapeutic team. The latter would have a coordinator (who might be a member of the CMHT or a visiting member of the PTU) with its own waiting list. Each member of the team would have defined number of treatment 'slots', thereby limiting the mental health worker's case load. All cases would be supervised, audited and members would participate in skills acquisition, reading seminars, case presentation etc.

\section{Care pathways in psychotherapy}

The progress of each referral can be tracked along a number of possible routes, or 'care pathways'.

Patients suffering from mild depression referred by their general practitioner, will, after assessment, be allocated to the brief intervention division within the CMHT. After waiting until a 'slot' arises they could be offered a timelimited self-esteem group run along CBT lines by two members of the CMHT team, supervised by the visiting CBT therapist from the PTU. Those whose symptoms have remitted to some extent, but who continue to have major interpersonal difficulties making them vulnerable to further episodes of depression, will at this point be referred on to the PTU, where, following assessment, they may be allocated to longerterm treatment, perhaps in a group run along group analytic lines, managed by a qualified therapist.

More complex cases, say of severe personality disorder, would after initial screening by the CMHT, be referred directly to the personality disorder service which is part of the PTU, and be offered longer-term treatment from the start of their contact with secondary services.

Patients suffering from a first episode of psychosis, perhaps following a period as an inpatient, will be taken into the CMHT and held supportively there until a 'slot' arises in the psychosis intervention programme. They would then be offered intensive CBT and family therapy, under the supervision and auspices of a member of the psychosis intervention team from the PTU, although the therapists will be members of the CMHT. At the completion of 
treatment (perhaps after 6-9 months) the patient will be allocated to routine supportive holding under CPA with a keyworker within the CMHT.

\section{Conclusion: management and brokerage}

The purpose of this paper has been to describe ways in which psychotherapeutic treatments can become part of routine psychiatric care. The evidence for the efficacy of such treatment exists (Roth \& Fonagy, 1996), and there is a growing body of knowledge about the economic as well as clinical advantages of combining pharmacological and psychological therapies (Gabbard et al, 1997). It still remains to convert research evidence and clinical enthusiasm into everyday practice. Given the strength of its evidencebase it is scandalous if psychotherapy is to remain a luxury to which patients only have access if they happen to live in a favoured area, or can afford private treatment (Holmes \& Lindley, 1998).

The key to developing such services lies in the relationship between the PTU and CMHT. Shaping this relationship is a delicate management task, involving as it does the sensitivities and professional identities of general psychiatrists, psychologists, medical psychotherapists and psychiatric nurses. The necessary "brokerage' between them is likely to come initially from within the PTU, and will stand or fall depending on the relationship between the consultant psychotherapist and his general psychiatric colleagues (Working paper from the Psychotherapy Faculty of the College: The development of psychotherapy services: the role of the consultant psychotherapist'; further details available from the author upon request). Medically qualified consultant psychotherapists combine psychotherapeutic and psychiatric expertise, and should be accepted culture-carriers in both fields.

Mental health units and commissioning bodies wishing to provide equitable, evidence-based mental health services that are effective and acceptable to the public would do well to encourage such brokerage. Creating and supporting consultant psychotherapist posts is an essential part of that process.

\section{Acknowledgements}

The ideas in this paper have emerged from discussions with my colleagues: in the Psychotherapy unit at North Devon, especially Mrs Mary Marshman and Dr Glenn Roberts; in Bristol with Drs David Whitwell, Judy Malone and Andrew Clark; and in the Psychotherapy Faculty of the Royal College of Psychiatrists, especially Drs Michael Hobbs, Frank Margison, Bernard Roberts and Anthony Bateman.

\section{References}

Albeniz, A. \& Holmes, J. (1995) Integration in psychotherapy: implications for psychiatry. British Journal of Psychiatry. 169, 563-570.

BATEMAN, A. (1997) Borderline personality disorder and psychotherapeutic psychiatry. British Journal of Psychotherapy. 13, 489-498.

GABBARD, C.. LAzAR, S., HORBERger, P., et al (1997) The economic impact of psychotherapy: a review. American Journal of Psychiatry. 164, 147-155.

HoLMES, J. \& MrTCHISON, S. (1995) A model for an integrated psychotherapy service. Psychiatric Bulletin, 19, 210213.

- \& LINDLEY, R. (1998) The Values of Psychotherapy (2nd edn). London: Karnac.

National Health Service ExfCutive (1994) Guidance on the Discharge of Mentally Disordered People and their Continuing Care in the Community. London: HMSO.

- (1996) Psychotherapy Services in England. London: HMSO.

RoTH. A. \& FonAGY, P. (1996) What Works for Whom. New York: Guilford Press.

ROYAL COLLEGE OF PSYCHIATRISTS (1991) The future of psychotherapy services. Psychiatric Bulletin, 16. 174179.

Jeremy Holmes, Department of Psychiatry, North Devon District Hospital, Barnstaple, Devon EX31 $4 J B$ 